\title{
Cases of Leptospirosis after typhoon Ondoy (international name: Ketsana) seen at the emergency department: the Philippine General Hospital experience
}

\author{
A. Balbuena, T. Herbosa \& C. P. Gundran \\ Department of Emergency Medicine, University of the \\ Philippines-Philippine General Hospital, The Philippines
}

\begin{abstract}
Emergency care physicians are at the forefront of receiving patients in conditions of widespread flooding such as what happened when Typhoon Ondoy poured heavy rain on September 26, 2009 and brought the worst rainfall to Metro Manila among recorded typhoons according to PAGASA (Philippine Atmospheric, Geophysical, and Astronomical Services Administration). Luzon was declared in a state of calamity. Flood water levels reached a record 20 feet high in rural areas. More than 2,089 people have been treated for bacterial infection in Manila and surrounding provinces and 162 people have died, more than five times the number of Leptospirosis deaths in the entire country in 2008.

This study is a chart review of the clinical outcome of admitted patients diagnosed with Leptospirosis during Typhoon Ondoy from October to November 2009. This covered those patients' charts designated under the ICD (International Classification of Disease) A27 and showed their clinical outcome based on the following predictors such as age, gender, duration of hospitalization, presence or absence of complications particularly bleeding diathesis, renal failure, cardiac, neurologic and muscular complications on admission.

The majority of mortalities is associated with bleeding diathesis. Cardiac involvement is common but may be underestimated. Having in mind the complications of Leptospirosis and predictors of mortality, early detection and intervention, interim guidelines on management and public health measures can respond better to expected cases especially during an epidemic/disaster.
\end{abstract}

Keywords: typhoon Ondoy, Ketsana, Leptospirosis, bleeding diathesis renal failure, pulmonary hemorrhage. 


\section{Introduction}

Leptospirosis (mud fever/ swamp fever) is a disease most often unrecognized [13]. It affects both humans and animals. Its protean manifestations simulate various systemic conditions involving renal, hepatic and hemorrhagic problems after the organism enters the body when mucous membranes or abraded skin come in contact directly or indirectly with contaminated environmental sources. They multiply in the bloodstream and spread to any part of the body $[2,13]$.

Leptospirosis is spread epidemically in conditions of widespread flooding following a typhoon or heavy rain when rats are driven out of sewers. Such an incidence of Leptospirosis occurred in the Veneto area in Northeast Italy [1] and in Nicaragua in 1995 [7]. In 2004, Leptospirosis cases were found among those involved in the clean-up process when a stream overflowed and caused flooding in a university campus [11]. The rat population is a risk since they carry within their kidneys the bacteria and excrete in their urine. Any exposure to water contaminated with their urine can spread the infection.

Leptospirosis was first reported in the Philippines in 1932. An estimated 147 cases per 100,000 population was noted in a 2007 local publication. In 1932 to 1970, 1971 to 1973 and 1998 to 2001 , there were sixty five (65), three hundred ninety (390) and eight hundred forty (840) respective cases documented in literature [15]. Contrary to the recent updates that there was a decreasing number of Leptospirosis in the Philippines, the recent outbreak belies that fact. There are over 200 serovars [12]. Leptospira interrogans is the most common agent and Leptopsira icterohemorrhagica, commonly associated with bleeding tendencies have been demonstrated among patients in the Manila area: and the other serovars are the following: poi, tarassovi, manilai, pyrogenes, australis, grippotyphosa, javanica and automnalis [15].

Flood-prone areas of urban settings such as Metro Manila were overwhelmed with cases of Leptospirosis after typhoon Ondoy/Ketsana. It is important for the clinician and the general population to know what to anticipate in such times since the disease is not often recognized due to a wide variety of clinical manifestations. Serological tests are diagnostic but is not always available especially in underdeveloped and developing countries. Therefore, earlier detection and intervention is necessary.

The main objective of the study was to determine the prevalence of Leptospirosis cases during typhoon Ondoy seen at the emergency department of the Philippine General Hospital from October to November 2009. Additional objectives were to determine the demographic characteristics as to age group and sex distribution of cases who survived and those who did not as well as present the morbidity and mortality rates based on chief complaint and duration of symptoms prior to admission; the degree of thrombocytopenia and the clinical manifestations such as renal failure, bleeding diathesis, musculoskeletal, cardiac and neurological manifestations in relation to their outcome. 


\section{Materials and methods}

\subsection{Study design, population and data source}

This was a retrospective descriptive study in all patients diagnosed to have Leptospirosis admitted at the emergency room from October 1 to November 30, 2009. The data source are the statistical records kept by the University of the Philippines-Philippine General Hospital (UP-PGH) Medical Records with a diagnosis of Leptospirosis based on ICD (international classification of diseases) A27 and with PIC (Please Issue Chart) form under the ACU (Acute Care Unit) or PER (pedia ER). A total of 143 charts were reviewed.

\subsection{Analysis}

The study was conducted at the emergency room of the Philippine General Hospital of suspected cases based on initial clinical diagnosis using "The UPPGH Interim Guideline for the management of Leptospirosis" and eventually classified using ICD A27. The following data were collected using a data intake sheet which includes: age, sex, presenting complaint, duration of symptom before admission, clinical manifestations, complications, duration of hospital stay and severity of thrombocytopenia. By applying Yate's chi square test this study has shown the significance of predictors to the mortality of patients admitted with Leptospirosis.

\section{Results and discussion}

\subsection{Results}

The distribution of cases according to age and sex is shown in Figure 1. A total of 143 patients were included in the analysis. The youngest patient was a 9 year

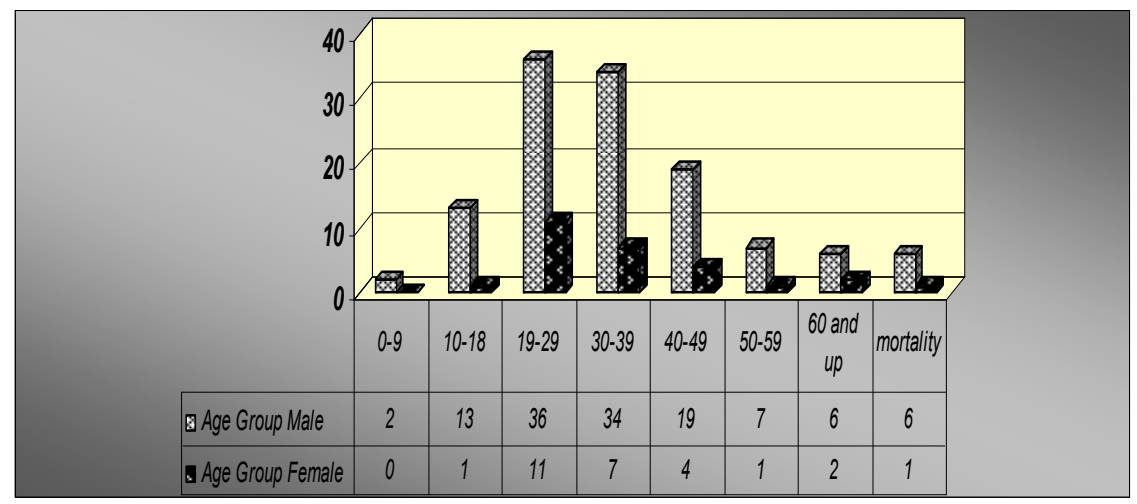

Figure 1: $\quad$ Age and sex incidence. 
old male while the oldest was a 71 year old male. There is male predominance of $81 \%$ (117 cases). The majority are in the age range of $19-39$ years old $(27 \%)$. Although over all mortality is relatively low (4.9\%) in both sexes $(4.20 \%$ male, $0.70 \%$ female), it is not due to sex-linked susceptibility (Yate's $\chi^{2}: 0.052, p$ $<0.05$ ) but rather due to their engagement in outdoor exposure to infected urine or indirect contact with contaminated water/flood in comparison to the female population.

Jaundice was the most usual complaint (39.8\%) with dyspnea (33.5\%) and fever $(18.1 \%)$ as second and third respectively as shown in Figure 2. Jaundice and dyspnea had the most number of complaints for mortalities at $30 \%$ each. Chest pain was seen in both groups with $9.8 \%$ mortality. Other presenting complaints were oliguria/anuria $(11.8 \%)$, calf pain $(2.8 \%)$, myalgia $(2.8 \%)$, headache $(4.9 \%)$, diarrhea $(2 \%)$, abdominal pain $(5.6 \%)$, vomiting $(3.5 \%)$, convulsions $(0.7 \%)$, behavioural changes $(0.7 \%)$.

The comparison of patients admitted $\leq 5$ days during the acute septicemic phase which presents with mild to moderate illness based on the interim

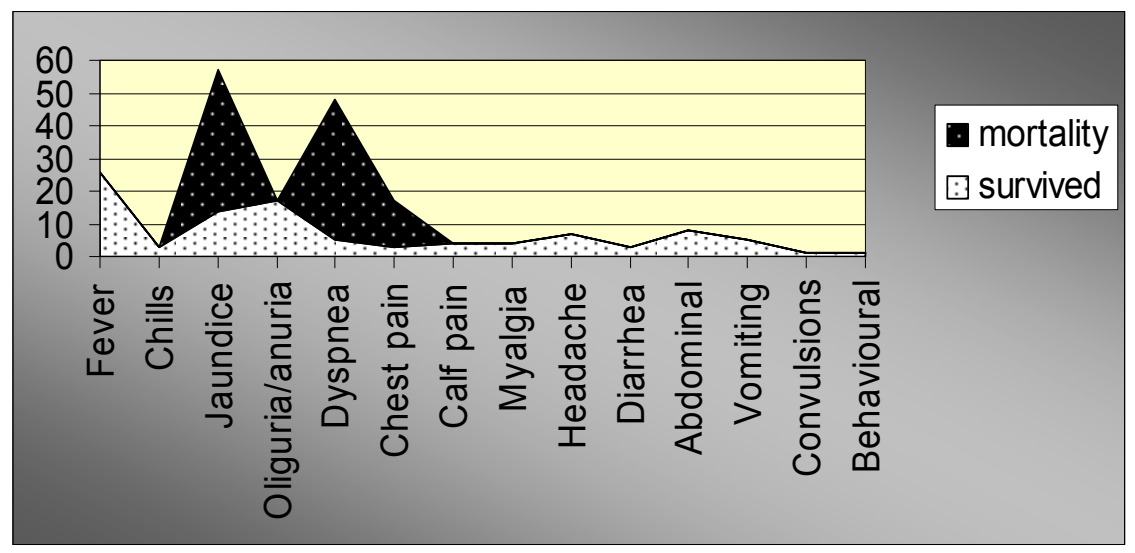

Figure 2: Chief complaint on admission.

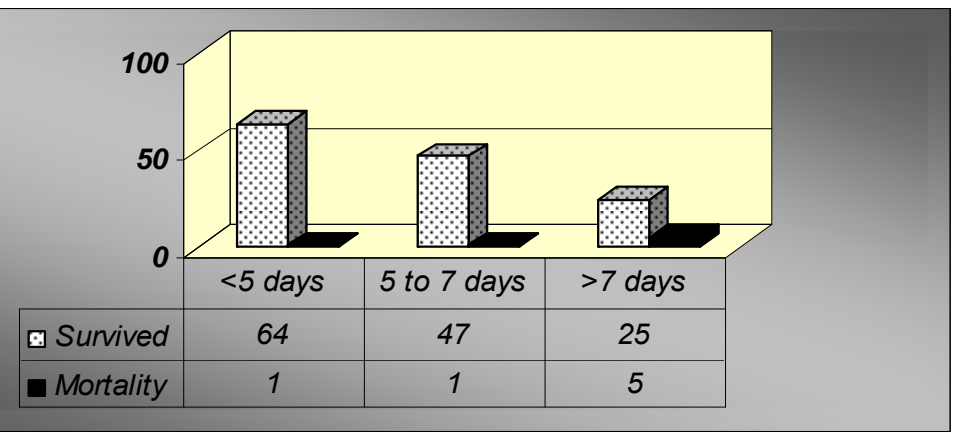

Figure 3: Duration of symptoms prior to admission. 
guidelines for management of Leptospirosis at the UP-PGH and those $>7$ days during the immune phase presenting with moderately to severely ill condition is shown in Figure 3. More than forty-four percent (44\%) with symptoms of less than 5 days survived. The duration of symptoms prior to consultation of two cases reported mortalities of less than a week (1.4\%). The majority of mortalities, however, were already presenting with symptoms of the disease a week or more $(3.5 \%)$. We tested the null hypothesis if the duration of symptoms prior to admission is independent of mortality (Yate's $\chi^{2}: 7.838, p>0.05$ ) and we reject the null.

The figure below (Figure 4) shows the duration of hospitalization of cases. The mean duration of hospitalization is 48 hours. There was only 1 mortality beyond 48 hours of admission ( $0.7 \%)$ and 6 mortalities were less than 48 hours after admission. The majority were confined for up to 48 hours (94\%). The null hypothesis proved the duration of hospitalization is not a significant predictor of mortality (Yate's $\chi^{2}: 1.739, p<010$ ).

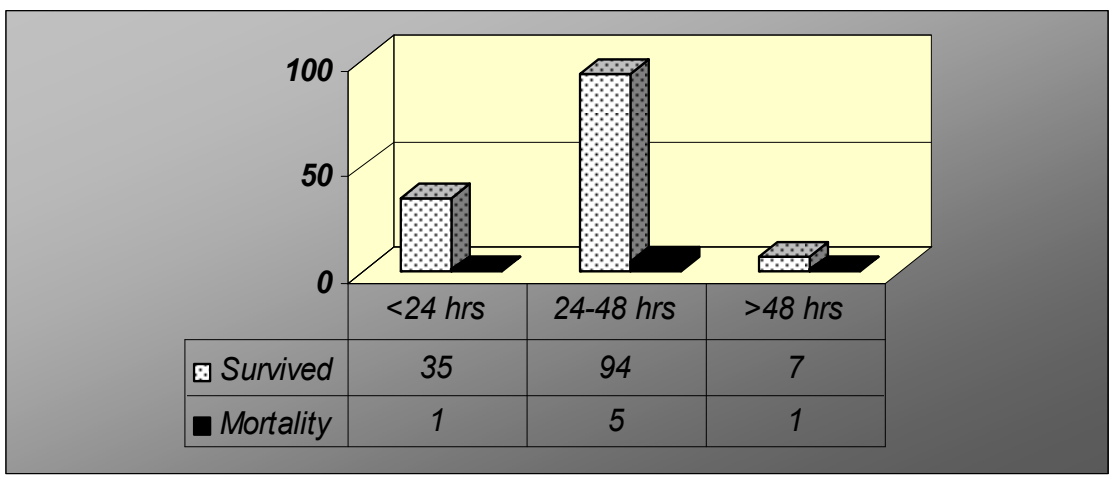

Figure 4: $\quad$ Duration of hospitalization.

Figure 5 describes the outcome of patients based on their clinical manifestations. Though upper gastrointestinal bleeding, hemoptysis, melena, intracranial bleed, presence of petechiae/ecchymoses which comprise those who survived seen in bleeding diathesis (40\%), majority died also of bleeding complications manifested as a pulmonary hemorrhage (71\%) which is a significant predictor of mortality when compared to the degree of thrombocytopenia Figure $6\left(\mathrm{x}^{2}: 20.118, \mathrm{p}<0.05\right)$. The prothrombin time was not used as a bleeding parameter since it was not determined in all patients. Twentyfive percent $(25 \%)$ presented with renal failure (azotemia, decreased urine output). Cardiac manifestations include chest pain, myocarditis, heart failure, arrythmias and comprise $9.79 \%$ and $2.09 \%$ for morbidity and mortality respectively $\left(\chi^{2}: 7.118, p>0.05\right)$. Non specific ST-T wave changes on electrocardiogram and those with pre-existing cardiac disease were excluded. Musculoskeletal manifestations include calf pain and myalgia which comprise $16 \%$. However, some of the charts reviewed do not include these in the review of 


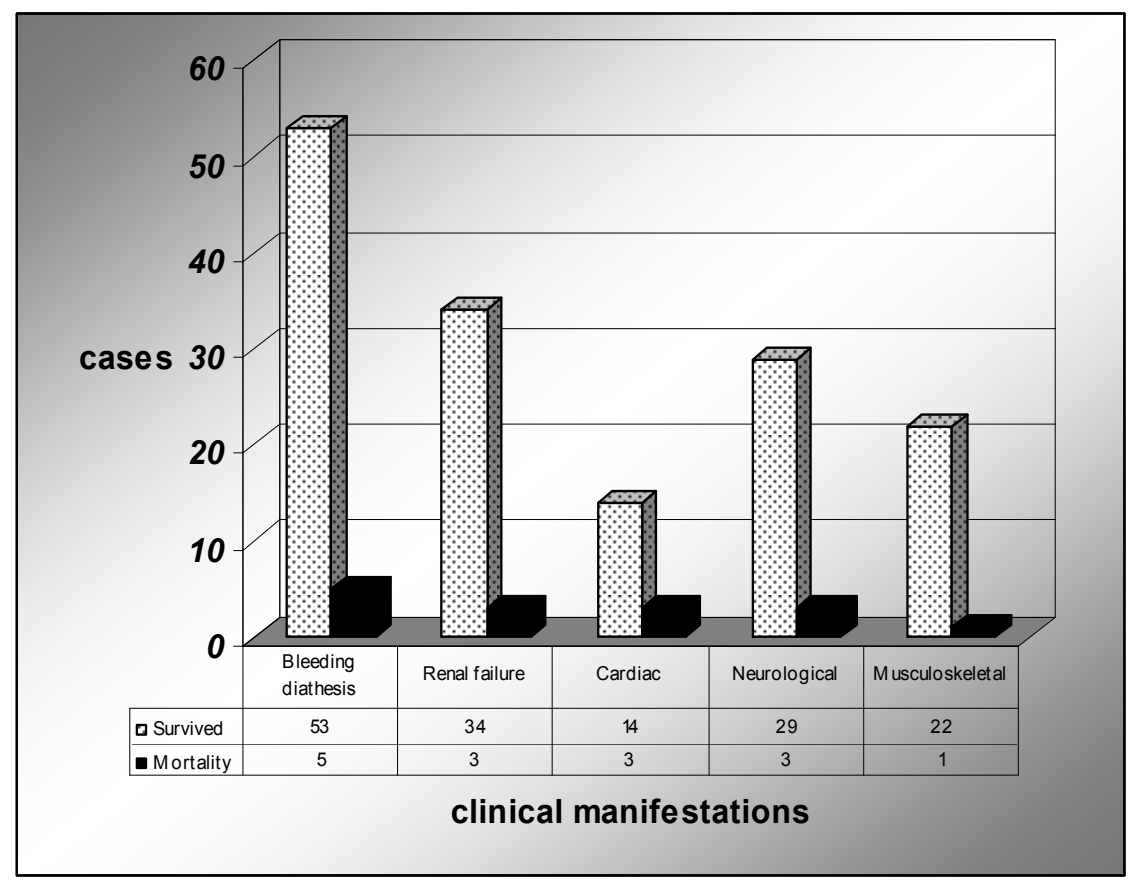

Figure 5: Clinical manifestations.

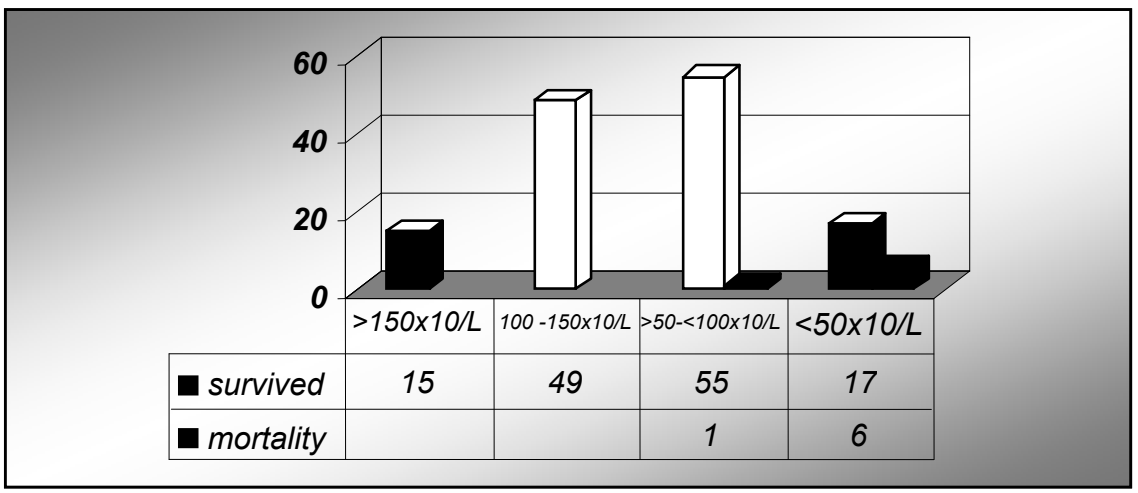

Figure 6: Severity of thrombocytopenia.

systems. Neurological complications include aseptic meningitis, intracranial hemorrhage shown as the third presenting clinical manifestation both in terms of morbidity and mortality.

The severity of thrombocytopenia is shown in Figure 6. There were 15 cases within normal limits $(10.5 \%)$. Forty-nine had more than a hundred but less than 150,000 platelet count $(34.3 \%)$ while fifty-five cases $(38.5 \%)$ had more than fifty 
but below 100,000 decreased count. Those with a platelet count of less than 50,000 are associated with increased mortality (Yate's $\chi^{2}: 15.92, p>0.05$ ).

\subsection{Conclusion and recommendations}

Leptospirosis cases that recover uneventfully constitute the majority in this study. Though self-limited, it leaves its mark on almost every organ system of the body by severe involvement of the liver, kidneys and vascular system which accounts for the majority of deaths. Of the 143 cases we studied, 7 died. All mortalities, except one, were serologically positive for Leptospira commonly associated with the classical Weil's disease. Five of them died of pulmonary hemorrhage and noted to be thrombocytopenic. One mortality was differentiated with Dengue Shock Syndrome but later on classified as ICD 27. The degree of hemorrhagic diathesis was associated with more fatal complications of pulmonary hemorrhage. This is possibly due to leptospiral vasculitis, undetected antiplatelet antibody, or to DIC (disseminated intravascular coagulation). There is a changing pattern of virulence of the disease and identifying the serovars involved would be most helpful. The unavailability of specific antigens for the different serovars made it difficult to rule out if it is a co-infection or not. It is important for clinicians to be aware and recognize the various clinical manifestations of Leptospira and be able to differentiate it from other acute febrile illnesses. Thrombocytopenia was associated with the occurrence of bleeding manifestations and therefore its severity is a predictor of mortality. In addition, the duration of symptoms prior to admission, clinical manifestations and its complications contribute to the mortality of the patient. The possibility of other infectious agents mimicking the presentation of Leptospirosis should be entertained. No relation between drug use and recovery was established since the virulence of the organisms and certain host factors determine the outcome. Different treatment modalities in terms of antibiotic use were received by patients initially and uniformity came later when an interim guideline on management was released. It is therefore recommended that future undertakings include study on treatment and the availability of practice guidelines to better manage that the patient be available.

\section{References}

[1] Pellizzer P, Todescato A, Benedetti P, Colussi P, Conz P, Cinco M. Leptospirosis following a flood in the Veneto area, North-east Italy. Ann Ig.,pp.18(5):453-6, 2006.

[2] Palaniappan RU, Ramanujam S, Chang YF. Leptospirosis: pathogenesis, immunity, and diagnosis. Curr Opin Infect Dis.;20(3):284-92, 2007.

[3] Inada R, Ido Y, et al. Etiology, mode of infection and specific therapy of Weil's disease. J Exp Med. 1916; 23:377-402.

[4] CDC. From the Centers for Disease Control and Prevention. Update: outbreak of acute febrile illness among athletes participating in Eco- 
Challenge-Sabah 2000--Borneo, Malaysia, 2000. JAMA; 285(6):728-30, 2001.

[5] CDC. Update: Leptospirosis and unexplained acute febrile illness among athletes participating in triathlons--Illinois and Wisconsin, 1998. MMWR Morb Mortal Wkly Rep.; 47(32):673-6, 1998.

[6] Sulit Y. Features of Weil's Disease. A review of cases in the Philippine General Hospital with comments from the literature. Acta Medica Philippina 1963; 19(4):196.

[7] CDC. Outbreak of acute febrile illness and pulmonary hemorrhage-Nicaragua, 1995. JAMA;274(21):1668, 1995.

[8] Gaynor K, Katz AR, Park SY, Nakata M, Clark TA, Effler PV. Leptospirosis on Oahu: an outbreak associated with flooding of a university campus. Am J Trop Med Hyg.; 76(5):882-5, 2007.

[9] Cacciapuoti B, Ciceroni L, Maffei C. A waterborne outbreak of leptospirosis. Am J Epidemiol; 126(3):535-45, 1987.

[10] Carvalho CR, Bethlem EP. Pulmonary complications of leptospirosis. Clin Chest Med.; 23(2):469-78, 2002.

[11] CDC. Brief report: Leptospirosis after flooding of a university campus-Hawaii, 2004. MMWR Morb Mortal Wkly Rep; 55(5):125-7, 2006.

[12] Human Leptospirosis: Guidance for Diagnosis, Surveillance and Control World Health Organization, 2003.

[13] Green-McKenzie J, Shoff W, Leptospirosis in Humans, emed specialties Department of Emergency Medicine, University of Pennsylvania School of Medicine, University Hospital

[14] Griffith ME, Hospenthal DR, Murray CK. Antimicrobial therapy of leptospirosis. Curr Opin Infect Dis.; 19(6):533-7, 2006.

[15] Gideon Informatics, Leptospirosis in the Philippines, www.GIDEONonline.com

[16] Takafuji ET, Kirkpatrick JW, Miller RN, et al. Agricultural Occupational Medicine: An Efficacy Trial of Doxycycline Chemoprophylaxis against Leptospirosis. In: Occupational Medicine. St Louis, MO: Mosby; 310, 497500, 894-5,1984

[17] Preacher, K. J. Calculation for the chi-square test: An interactive calculation tool for chi-square tests of goodness of fit and independence [Computer software]. http://www.quantpsy.org/. 